\title{
Pneumoscrotum as Complication of Blunt Thoracic Trauma: A Case Report
}

\author{
Patel Ravisagar ${ }^{1}$, Singh Abhinav ${ }^{2}$, Devgarha $\mathrm{S}^{3}$, Mathur $\mathrm{RM}^{4}$ \\ ${ }_{1,2,3,4}$ (Department of cardiothoracic and vascular surgery, SMS medical college, Jaipur India)
}

\begin{abstract}
Pneumoscrotum following traumatic pneumothorax is a rare clinical scenario. We report the case of a 62-year-old male who presented to the Cardiothoracic Department with respiratory distress sustaining blunt thoracic trauma following RTA. Massive surgical emphysema was present,extending from the face upto the scrotum. B/L chest tube were inserted with immediate gross improvement of the vital parameters of the patient.
\end{abstract}

Key words: camper's fascia, pneumomediastinum, pneumoscrotum, pneumothorax, scarpa's fascia

\section{Introduction}

Pneumoscrotum describes the presence of air within the scrotum .It includes scrotal emphysema when air is palpated as crepitus and scrotal pneumatocele when air is present within the tunica vaginalis testis but not palpable [1].

Air can derive from three different routes :a) extraperitoneal sources (retroperitoneum, mediastinum, or lungs), b) intraperitoneal sources (air-Filled hollow viscera), c) local sources (gas production due to infection or air introduction), and depending upon the cause appropriate treatment must be provided, especially in life threatening conditions such as pneumothorax or Fournier's gangrene.

\section{Case Report}

A 62 yr-old male patient presented with blunt trauma chest following road traffic accident. He had respiratory distress but was hemodynamically stable (BP: 144/80mmHg, HR: 110/min ,SpO2: 82\% on room air, RR: 34/min), with Glasgow Coma Scale of 15/15. The patient had abrasions on his forehead and on the right iliac fossa.

On systemic examination surgical emphysema extending from face to the scrotum was present "Fig 1 ". Tenderness was present over the right lower chest wall along with bony crepitus over the $5^{\text {th }}, 6^{\text {th }}, 7^{\text {th }}$, and $8^{\text {th }}$ rib .Auscultation of chest revealed crackling sound due to emphysema with $\mathrm{B} / \mathrm{L}$ decreased breath sounds.. He was a chronic smoker and known case of COPD

$\mathrm{X}$-ray chest revealed $\mathrm{B} / \mathrm{L}$ pneumothorax with fracture of $1^{\text {st }}$ to $8^{\text {th }}$ rib on the right side and $1^{\text {st }}$ to $7^{\text {th }}$ rib on the left side "Fig 2". ultrasound of the abdomen was normal, and CT scan of head was normal except for subcutaneous emphysema extending upto the temporal-parietal region. CT Scan of thorax confirmed the findings of $\mathrm{x}$ ray chest with pneumomediastinum "Fig 3". CT abdomen revealed no visceral or solid organ injury except for emphysema extending upto the scrotum.

B/L chest tubes were inserted. Patient was managed with oxygen supplementation, IV fluids, parenteral antibiotics and analgesics. B/L Pneumothorax and surgical emphysema gradually resolved. Patient was discharged in stable condition. He is asymptomatic and in regular follow up.

\section{Discussion}

Pneumoscrotum is a rare clinical manifestation. The main symptom is swelling of the scrotum, usually with no pain or tenderness [2] .The first case was described in 1912 following nephrotomy [3]. Since then, few cases have been reported caused either by procedural or pathological processes [1]. Pathologic pneumoscrotum includes pneumothorax (traumatic or spontaneous), pneumomediastinum, visceral perforation, trauma of the abdomen cavity or the retroperitoneum, direct scrotal injury, perinephric abscess, rapid decompression after diving, and Fournier's gangrene. Procedural causes of pneumoscrotum includes endoscopy and laparoscopic procedures [4, 5], retroperitoneal sympathectomy, pacemaker placement, intubation of the trachea, cardiopulmonary resuscitation.

Air accumulation in the scrotum can be explained by 3 pathophysiologic mechanisms.

A) gas-producing infection (i.e., Fournier's gangrene) or direct introduction of air (i.e., scrotal trauma).

B) presence of intra-abdominal air. In cases of pneumoperitoneum (usually from visceral perforation), air can reach the abdominal wall (by diffusion or through small peritoneal defects) and can accumulate in the scrotum dissecting along the fascial planes [6]. Sometimes, air can travel to the scrotal sac through a patent processus vaginalis [7].this embryologic defect is present in $15-30 \%$ of adults [8]. 
C) air spread from a thoracic source (pneumothorax or pneumomediastinum). In the case of pneumothorax, air travels from the lungs along the superficial subcutaneous fascia of Camper's and the deep membranous layer of Scarpa's fascia of the . abdomen .Fascial planes are distinct to the abdomen but fuse to form the Colles' fascia at the base of the penis and Dartos fascia in the testes, respectively [1,9]. It must be emphasized that pneumoscrotum itself is a benign condition thorough search must be done to rule out the underlying pathology .if pneumothorax be the cause placement of chest tube suffices and is life saving in deteriorating patients.

In our patient blunt trauma to the thorax resulted in bilateral pneumothorax that led to massive surgical emphysema which propagated to face through the neck and to the scrotum through the abdomen fasciae.

\section{Conclusion}

The aim of presentation of this case is that after blunt thoracic trauma one needs to be alert and aware when dealing with scrotal swelling that the primary source of air can be located in the thorax which may be managed by chest tube insertion.

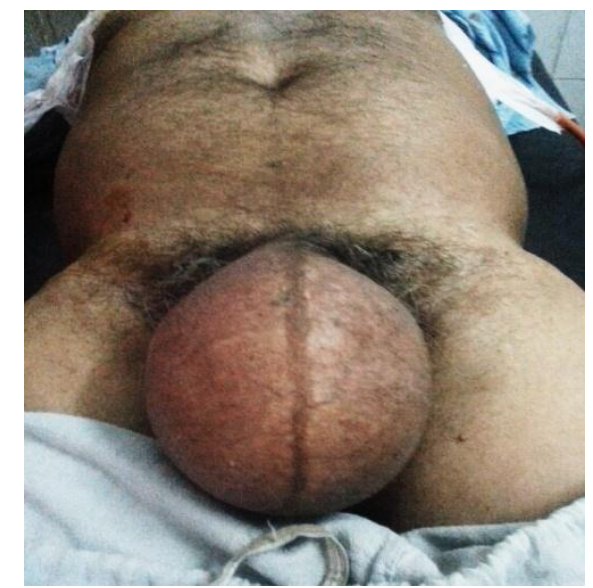

FIGURE 1 image showing bilateral ICD tubes and pneumoscrotum

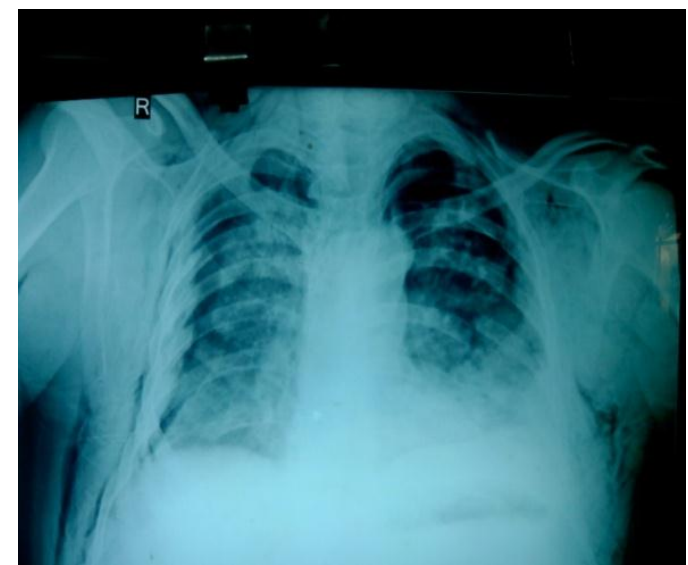

FIGURE 2 chest $\mathrm{x}$ ray showing bilateral pneumothorax with bilateral multiple rib fractures

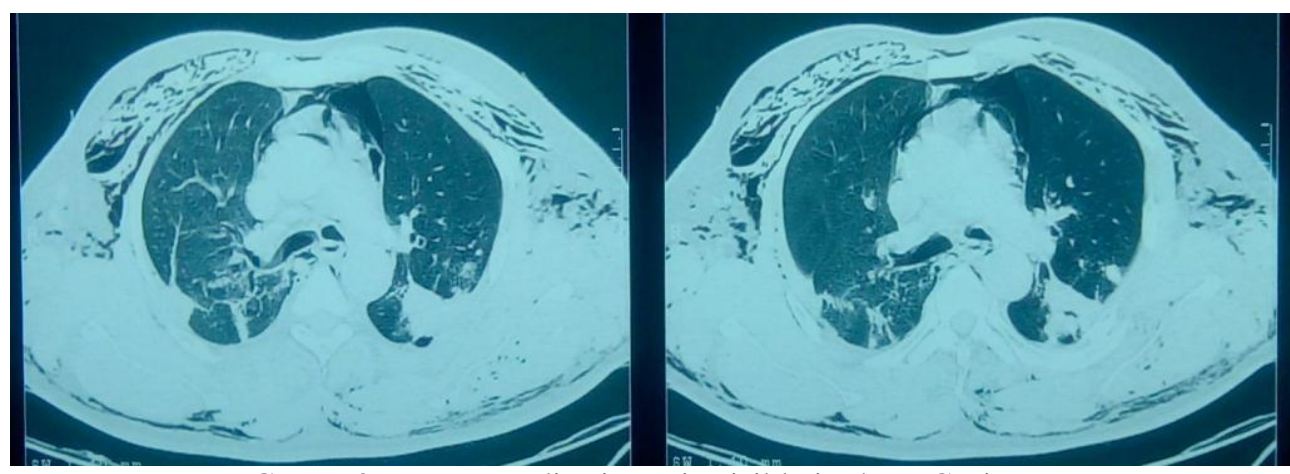

FIGURE 3 pneumomediastinum is visible in these CT images 


\section{References}

[1]. R. Firman, D. Heiselman, T. Lloyd, and P. Mardesich, "Pneumoscrotum," Annals of Emergency Medicine, vol. 22, no. 8, pp.13531356, 1993.

[2]. Y. Wakabayashi and W. H. Bush Jr., "Pneumoscrotum after blunt chest trauma," The Journal of Emergency Medicine, vol. 12, no. 5, pp. 603-605, 1994.

[3]. E. L. Keyes, "Two cases of pneumo-scrotum following nephrotomy," $\square$ e American Journal of Urology and Sexology, vol. 8, pp.68-71, 1912 .

[4]. R. Hasel, S. K. Arora, and D. R. Hickey, "Intraoperative complications of laparoscopic cholecystectomy," Canadian Journal ofAnaesthesia, vol. 40, no. 5 I, pp. 459-464, 1993.

[5]. N. Katkhouda, G. M. R. Campos, E. Mavor, A. Trussler, M. Khalil, and R. Stoppa, "Laparoscopic extraperitoneal inguinal hernia

[6]. repair: a safe approach based on the understanding of rectus sheath anatomy," Surgical Endoscopy, vol. 13, no. 12, pp. 1243-1246, 1999.

[7]. W. Mazraany, H. Kohli, A. Gabriel, G. R. Machado, M. O. Bojalian, and M. E. Reeves, "Massive pneumoscrotum following open

[8]. gastrostomy," Contemporary Surgery, vol. 60, pp. 219-221, 2004.

[9]. T. W. Hill, L. D. Mills, and C. J. Butts, "Pneumoscrotum after jejunal perforation: a case report," The Journal of Emergency Medicine, vol. 42, no. 3, pp. 279-282, 2009.

[10]. M. I. Rowe, L. W. Copelson, and H. W. Clatworthy, "The patent processus vaginalis and the inguinal hernia," Journal of Pediatric

[11]. Surgery, vol. 4, no. 1, pp. 102-107, 1969. R. S. Snell, "The abdomen: part I-the abdominal wall," in Clinical Anatomy by Regions, pp. 147-150, Lippincott Williams \& Wilkins, Baltimore, Pa, USA, 2007. 